\title{
Sandblasted by the Sun
}

\author{
The Earth's magnetic field protects us from solar activity, but the Moon and Mars are more exposed. The \\ upcoming solar maximum is the perfect time to observe how our dynamic Sun affects its planets.
}

Spectacular displays of the aurora borealis have graced the high northern latitudes this winter. Once interpreted in folklore as celestial omens, spirits or serpents, the northern and southern lights are now known to result from interactions between the solar wind and the Earth's magnetosphere. Charged particles that constitute the solar wind are accelerated polewards along the Earth's magnetic field lines and hit the electrically neutral atmosphere. On collision, atmospheric gases are excited and emit both light and heat to form the aurora. During periods of enhanced solar activity, such as in January this year when the strongest solar storm in seven years hit the Earth, the aurora reaches lower latitudes and can be observed more widely.

The recent burst of activity is probably only the beginning of a few geomagnetically tempestuous years. Solar activity rises and falls in an 11-year cycle due to variations of the Sun's magnetic field, and the next solar maximum is expected in 2013 . The near future is thus likely to bring more sunspots, solar flares and intense bursts of solar wind. The Earth is largely protected from the solar wind by its magnetic field, but not all planetary bodies are so lucky: both the Moon and Mars lack a shielding global magnetic field. The coming solar maximum presents a window of opportunity to study how the Sun's recurring activity affects these planetary environments.

With neither a magnetic nor an atmospheric shield, the Moon is particularly vulnerable to solar activity. According to simulations by NASA's DREAM (Dynamic Response of the Environment at the Moon) Centre, intense bursts of solar wind can erode the lunar surface like a sandblaster, because there is so little atmosphere to protect it (J. Geophys. Res. http://doi.org/fzc239; 2011). Plasma ions hitting the Moon can cause sputtering of atoms from the lunar surface. Also, simulations of solar wind interactions with lunar topography predict the diversion of solar wind ions towards polar crater floors, which has important implications for the formation and depletion of volatile reservoirs in the Moon's shadowed polar regions (Geophys. Res. Lett. 38, L19202; 2011).

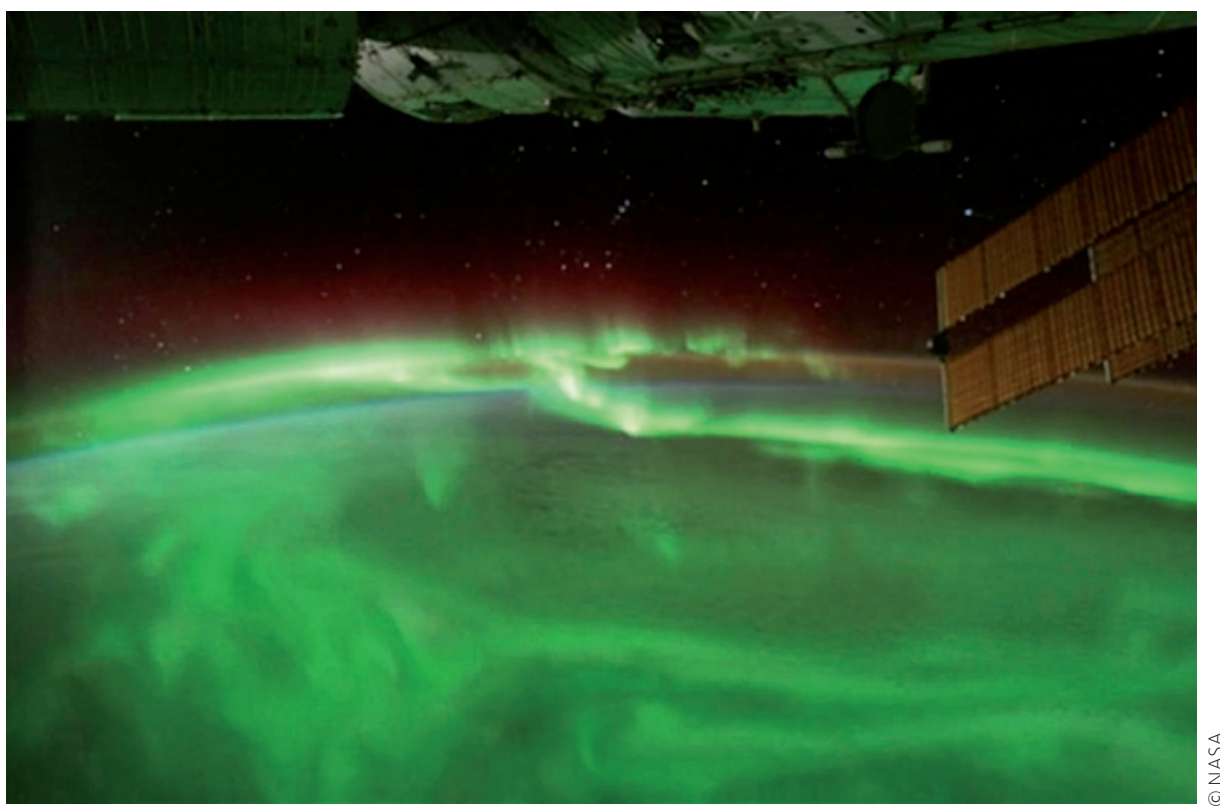

These predictions from the DREAM team simulations will be tested during the upcoming solar maximum by two NASA missions: ARTEMIS (Acceleration, Reconnection, Turbulence and Electrodynamics of the Moon's Interaction with the Sun) and LADEE (Lunar Atmosphere and Dust Environment Explorer). The two spacecraft assigned to the first project are already in orbit around the Moon and are studying how the solar wind passes over it. The second mission is scheduled to launch in 2013 and will closely observe the near-surface lunar environment at an altitude of 20 to $50 \mathrm{~km}$. It is expected that during solar storms, lunar surface atoms will be sputtered to LADEE's orbital altitude where the spacecraft can observe them.

Mars also lacks a global magnetic field, but its surface is protected by a thin atmosphere. However, the sputtering effect that erodes the lunar surface may affect the martian atmosphere in a similar way. The upper atmosphere of Mars is directly exposed to the solar wind, and atmospheric erosion, normally at a low background level of $<1 \mathrm{~kg} \mathrm{~s}^{-1}$ (Nature 341, 609-612; 1989; Science 26, 501-503; 2007), is expected to rise during enhanced solar activity. For example, high rates of ion fluxes away from the martian atmosphere were documented during a solar storm during the last solar minimum by the Mars Express mission (Planet. Space Sci. 56, 873-880; 2008; Geophys. Res. Lett. 37, L03107; 2010). These data can be compared with observations during the solar maximum from NASA's MAVEN (Mars Atmosphere and Volatile Evolution) mission, scheduled to launch in 2013 to study how the how the martian upper atmosphere interacts with the solar wind. The combination of data should bring more comprehensive insights into how martian atmospheric erosion varies across the 11-year solar cycle - and how Mars lost its once wet, thick atmosphere.

The increase in space weather over the coming months will affect all the planets. Like the Earth, Jupiter and Saturn do have global magnetic fields and will probably experience enhanced auroral activity. Although the Earth's surface is largely shielded, the poles and man-made systems in space are not. As we head towards the next solar maximum, the Earth is likely to experience more geomagnetic storms that may disrupt satellite and communications systems, as well as flight routes. Prediction and close observation of space weather will be essential for understanding how our Sun influences near-surface planetary environments. 